\title{
INVESTIGACIÓN/RESEARCH
}

Recibido: 18/09/2012---Revisado: 16/09/2012 Aceptado: 23/12/2012---Publicado: 15/07/2013

\section{EL TRABAJO DE CAMPO COMO ESTRATEGIA PEDAGÓGICA INTEGRADORA}

René Delgado La Rosa1: Universidad Pedagógica Experimental Libertador. Venezuela. renedlgd@yahoo.com.mx

\section{RESUMEN}

El presente estudio tuvo como finalidad analizar la experiencia del trabajo de campo como estrategia pedagógica integradora desarrollada con los estudiantes de los cursos Metodología de la Educación Integral I y II de la especialidad de Educación Integral en el Instituto Pedagógico de Miranda José Manuel Siso Martínez. La metodología utilizada se corresponde con el paradigma interpretativo con un enfoque cualitativo de carácter descriptivo, bajo la modalidad de campo. Las conclusiones más resaltantes fueron: (a) El trabajo de campo constituye una estrategia pedagógica para la Educación Primaria que permite integrar los contenidos disciplinares y científicos, los ejes transversales y los contenidos sociales - contextuales. (b) El uso de los enfoques geohistórico y globalizador permitió trabajar didácticamente desde lo contextual y local para el entendimiento del fenómeno o problema con una visión social, global y compleja. (c) Se identificaron algunas dimensiones caracterizadoras del trabajo de campo, a saber: social comunitaria; didáctico - pedagógica; cognitiva - constructiva; ecológica - ambiental; científico - académica; exploratoria - investigativa, recreativa y valorativa.

PALABRAS CLAVE: Trabajo de Campo - Estrategia Pedagógica - Currículo Integrado.

\footnotetext{
${ }^{1}$ Autor correspondiente:

René Delgado La Rosa: Departamento de Práctica Profesional del Instituto Pedagógico de Miranda José Manuel Siso Martínez de la Universidad Pedagógica Experimental Libertador. Caracas. Venezuela.

Correo: renedlgd@yahoo.com.mx
} 


\title{
THE FIELD WORK AS AN INTEGRATIVE PEDAGOGICAL STRATEGY
}

\begin{abstract}
The present study aimed to analyze the experience of fieldwork as an integrative teaching strategy developed with students of the Integral Education Methodology I and II courses of the Integral Education specialty at the Instituto Pedagógico de Miranda José Manuel Siso Martínez. The methodology is consistent with the interpretive paradigm with a descriptive qualitative approach, under the Field mode. The most striking findings were: (a) The field work is a pedagogical strategy for primary education which allows the integration of the scientific and disciplinary content, and crosscutting social - contextual content. (b) The use of geo-historical and globalizing approaches allowed the didactic work from the local context and understanding of the phenomenon or problem with a social vision, global and complex. (c) Some dimensions characterizing the field work was identified, namely- community, teaching - teaching, cognitive - constructive ecological environmental science - academic, exploratory - research, recreational and valuation.
\end{abstract}

KEYWORDS: Fieldwork - Pedagogical Strategy - Integrated Curriculum.

\section{INTRODUCCIÓN}

En la Universidad Pedagógica Experimental Libertador, específicamente en el Instituto Pedagógico José Manuel Siso Martínez, se administran los cursos Metodología de la Educación Integral I (MEI0714, UPEL, 1999a) y Metodología de la Educación Integral II (MEI0724, UPEL, 1999b) pertenecientes al plan de estudios de la especialidad de Educación Integral, particularmente al Componente de Formación Especializada, en el Área de Didáctica de la Especialidad del Departamento de Práctica Profesional.

Dicha administración se hace atendiendo a los objetivos propuestos en la especialidad (UPEL, 1997) en lo atinente a preparar a un docente con los conocimientos instrumentales necesarios; brindar formación en las áreas de Lengua, Matemática, Ciencias Sociales, Ciencias Naturales y Educación para el Trabajo, con un equilibrio entre la visión panorámica de la disciplina y el manejo especializado con uno o varios de sus sectores; desarrollar habilidades y actitudes favorables hacia la investigación permanente para incorporar nuevos conocimientos a fin de enriquecer e innovar su acción educativa; desarrollar habilidades, destrezas y actitudes para el manejo eficiente de la didáctica de procesos; formar profesores creativos que desarrollen habilidades para utilizar recursos didácticos variados; entre otros.

Asimismo, responden al perfil académico de Educación Integral planteado en el Diseño Curricular de la Especialidad (UPEL, 1997), en el cual se menciona que el estudiante adquiere una visión globalizadora del conocimiento que le permite establecer las 
relaciones, los diferentes grados de complejidad y la necesaria complementariedad ente las disciplinas que traten de explicarlo; domina en forma teórica - práctica las áreas del conocimiento en las que se inscriben los programas de Educación Básica (actualmente Educación Primaria).

Desde esta visión panorámica, el curso Metodología de la Educación Integral I, se constituye en una materia básica obligatoria que permite desarrollar las competencias para generar y aplicar estrategias pedagógicas que integran los conocimientos de las diferentes áreas. Mientras que el curso Metodología de la Educación Integral II guía su acción hacia la elaboración de los proyectos educativos en atención a los aspectos teóricos metodológicos que orientan su construcción; a situaciones reales concretas que se presentan en la escuela; al desarrollo evolutivo del niño; necesidades e intereses del educando; fines y objetivos de la educación primaria, perfil del egresado; ejes transversales y contenidos; competencias básicas a desarrollar en cada grado.

De allí que se plantea la necesidad de desarrollar un currículo integrado haciendo uso de estrategias pedagógicas integradoras y de proyectos educativos que permitan viabilizar el entendimiento de los fenómenos y problemas sociales y naturales, la construcción y reconstrucción del conocimiento, la contrastación de la teórico con lo práctico, etc. Bajo esta perspectiva se seleccionó el trabajo de campo como una de las posibles experiencias que permitiesen dar respuestas a tales exigencias curriculares y demandas educativas.

El trabajo de campo siempre se ha considerado como una actividad, empleada básicamente en el nivel universitario, para corroborar el aprendizaje o evidenciar in situ algunos fenómenos, situaciones o hechos vinculados con las ciencias sociales y áreas afines. Sin embargo, se argumenta en este artículo que se constituye en una estrategia de primer orden para la Educación Primaria pues permite la construcción y reconstrucción del conocimiento de manera global sobre un fenómeno o situación problemática; de contrastar situacionalmente la teoría con la práctica y de integrar los contenidos de un área y distintas áreas del conocimiento. En este sentido, se propone un análisis de la experiencia del trabajo de campo como estrategia pedagógica integradora, a través de los enfoques geohistórico y globalizador, que se desarrolló con los estudiantes de los cursos Metodología de la Educación Integral I y II de la especialidad de Educación Integral desde el Período Académico 2005 - I hasta el 2012 - I en el Instituto Pedagógico de Miranda José Manuel Siso Martínez (IPMJMSM) de la Universidad Pedagógica Experimental Libertador (UPEL). De lo que se trató fue de interpretar el proceso de integración de los contenidos disciplinares con el conocimiento contextual local; así como también, identificar las dimensiones que lo caracterizan desde estos enfoques. 


\section{METODOLOGÍA}

La metodología utilizada se corresponde con el paradigma interpretativo con un enfoque cualitativo, bajo la modalidad de campo de tipo investigación sobre la práctica (UPEL, 2003) y un diseño no experimental de carácter descriptivo. Los sujetos informantes que participaron en el estudio fueron escogidos de manera intencional en cada período académico de acuerdo con la actitud mostrada antes de la aplicación del trabajo de campo y los conocimientos previos de diferentes cursos de la especialidad.

Para llevar a cabo la investigación fue necesaria la aplicación de las técnicas de la observación y de la entrevista. En la primera técnica, se empleó el instrumento para la mediación cognitiva (Costa y Garmston, 1994) en la cual se consideraron tres aspectos relevantes: la planificación de la observación, la observación de la estrategia y la reflexión sobre la observación y; en la segunda, se utilizó un guion de entrevista constituido inicialmente por 8 preguntas divergentes. Además, fue necesaria la utilización de una hoja de observación semi-estructurada en segmentos de actividad formativa de acuerdo con la planificación realizada previamente. Los datos obtenidos fueron triangulados en función de las diferentes fuentes, técnicas y teorías; estos permitieron obtener categorías axiales y selectivas cuyo tratamiento interpretativo derivaron en el análisis que se presenta en este artículo.

Aunado a ello, se empleó una bitácora de trabajo que permitió orientar las actividades y tareas a realizar antes, durante y después de la aplicación de la estrategia. La misma se estructuró de la siguiente manera: (a) Bloqueo de la Zona: consistió en inspeccionar cada lugar donde se llevó a cabo el trabajo de campo. Durante el reconocimiento se delimitaron las áreas y el espacio de trabajo, así como también se planificó lo relacionado con la logística que fue discutida con los estudiantes del curso en cada período académico. (b) Plan de Trabajo: cuyo objetivo general se orientó a sensibilizar, monitorear y acompañar a los estudiantes del curso Metodología de la Educación Integral I y Metodología de la Educación Integral II del período académico respectivo en el proceso de aplicación del enfoque globalizador a fin de contrastar en la práctica la integración de los contenidos de un área y de diversas áreas del conocimiento a través de la aplicación de estrategias pedagógicas variadas en ambientes no convencionales. Para la consecución del objetivo se establecieron los siguientes objetivos específicos: 1 . Observar y comprender una realidad como un todo coherente, multidimensional, sistémico y complejo que permita una acción pedagógica integradora del conocimiento y del entendimiento de los problemas y en la búsqueda de soluciones viables y pertinentes. 2. Propiciar desde el trabajo de campo un espacio para la investigación desde la perspectiva inter y transdisciplinaria. 3. Poner en práctica variadas actividades pedagógicas en ambientes no convencionales desde un enfoque globalizador que atienda los contenidos de un área y de diversas áreas del conocimiento en el Nivel de Educación Básica. 4. Generar un espacio de dialogo y reflexión sobre el trabajo de campo como estrategia pedagógica integradora que permite la construcción y reconstrucción de los

Revista de Comunicación de la SEECI. (Julio 2013). Año XVII (30), 156-183 
saberes, la investigación, el trabajo cooperativo y el aprendizaje significativo haciendo uso del enfoque globalizador.

Las actividades generales se propusieron en función de cada grupo de trabajo para cada período académico, del cronograma previsto, en función del recorrido, de la zona y del contenido a trabajar. Aquí se presentan algunos ejemplos, a saber: (a) Revisión de las normas y dinámica de integración y de conformación de grupos. (b) Elaboración de canciones, gritos y consignas grupales. (c) Realización del diagnóstico situacional del espacio geográfico (descripción del contexto; ubicación del recorrido en el mapa respectivo haciendo uso de la brújula; identificación de zonas, sitios y edificaciones históricas; identificación de la Región y la relación entre relieve, clima, vegetación y fauna). (d) Elaboración del reloj solar. (e) Elaboración del diagnóstico de la localidad. Levantamiento de información a través de las técnicas observación y entrevista. (f) Elaboración de un mapa de recorrido en la localidad (mapa síntesis). (g) Elaboración de cuadrículas de área (determinación de medidas de superficie usando diferentes instrumentos de medición, recolección de muestras en superficie. identificación de plantas, animales y rocas). (h) Elaboración de estadísticos descriptivos. (i) Elaboración de dibujos de muestras recolectadas (observación, comparación y clasificación de las muestras según tamaño, color, forma, textura). (j) Determinación de áreas contaminadas (medición de áreas y tipos de desechos; determinación del PH en suelo, río o playa). (k) Aplicación de dinámicas de integración y socialización (elaboración de platos típicos con material del ambiente, propagandas vinculadas con el tema, etc.). (I) Circuito de Aprendizaje (ubicación de grupos de trabajo por postas correspondientes a las siguientes temáticas: integración de las comunidades a los proyectos de aprendizajes; integración de los conocimientos a través de la investigación y del trabajo de campo; el trabajo cooperativo y colaborativo; el contexto en el hecho pedagógico; el espacio geográfico y la cartografía). (m) Realización de plenaria y evaluación del trabajo de campo.

Asimismo, se emplearon algunos recursos didácticos y técnicos que permitieron el desarrollo de la estrategia, a saber: Mapas respectivos, filmadora, papeles de trabajo, cámaras fotográficas, cuaderno de notas, guía instruccional, medidor de $\mathrm{PH}$, altímetro, barómetro, entre otros.

Dentro de esta metodología, se parte del enfoque geohistórico en el encuentro de saberes entre las distintas disciplinas y las situaciones experienciales del ser humano para abordar un problema de interés o un fenómeno en específico y su influencia en el contexto local, sociocultural y en las personas. Ciertamente, se acepta el carácter social desde la dimensión témporo-espacial de las ciencias sociales, pero creemos que este enfoque adquiere otra dimensión al ser empleado conjuntamente con el enfoque dialéctico globalizador. Lo local e inmediato se estudia y comprende con lo global y distante; las ciencias sociales se funde con las ciencias naturales, la matemática, la literatura, las artes, el deporte y la recreación.

Revista de Comunicación de la SEECI. (Julio 2013). Año XVII (30), 156-183 
Particularmente, se emplean las tres herramientas propias del enfoque: diagnóstico de la comunidad, el método de los conjuntos y la cartografía conceptual (Sayago y León, 2000). La primera, permite caracterizar a la comunidad para estudiar los problemas observados y plantear algunas posibles soluciones, considerando la historia en el espacio y la relación hombre - comunidad - ambiente. En esta estrategia se toman en consideración: las características físico - naturales, el contexto histórico, el contexto educativo - cultural, la recreación y deporte, la salud, el contexto religioso y el culto, contexto político, el contexto socio - laboral y económico, contexto familiar (CENAMEC, s.f.b), y el contexto ambiental.

Con respecto al método de conjunto, permite la organización de la estructura de un espacio geográfico particular en una totalidad, identificando, desde lo interno, sus elementos constitutivos y el sistema de relaciones e interrelaciones atendiendo a la posición que guardan entre ellos (Sayago y León, op. cit.).

Este mismo autor afirma que la cartografía conceptual es una técnica didáctica que contribuye a representar y conceptualizar los elementos presentes en la realidad. En el cartograma se expresa, de manera gráfica y haciendo uso de códigos y símbolos, los elementos presente en la estructura espacial concreta y la acción de los grupos humanos, sus dinámicas y particularidades bajo las condiciones históricas producidas. Se emplea la técnica de la observación directa, las guías de información y de contexto espacial, un plano del área, hojas de registros, los registros fotográficos y los procesos de reflexión para captar el espacio geográfico tal cual como se presenta.

En algunas propuestas metodológicas la cartografía conceptual presenta la teoría del espacio abordado bajo unas categorías de análisis que son representadas en mapas síntesis. Éstos son recursos didácticos que reproduce un marco de nociones, conceptos y categorías del espacio, al indicar las condiciones precisas de localización de los elementos de dicho espacio (Palencia, 2003).

\section{ANÁLISIS Y DISCUSIÓN}

\subsection{Proceso de integración de los contenidos de las áreas disciplinares con el conocimiento contextual.}

Es necesario mencionar que los estudiantes de la especialidad Educación Integral tienen un perfil ocupacional dirigido a la Educación Primaria, es decir, van a ser profesionales que atenderán las exigencias y necesidades de los niños y adolescentes desde el primero al sexto grado, en edades comprendidas entre 6 y 12 años aproximadamente. En este sentido, como profesionales de la educación deben manejar y usar los programas respectivos de cada grado escolar; conocer el tratamiento adecuado para realizar la integración de los contenidos de la misma área y de las diferentes áreas académicas con 
los conocimientos contextuales; seleccionar y aplicar las estrategias pedagógicas apropiadas para el aprendizaje de los niños, entre otros. De allí, el hecho de incorporar un currículo integrado (Torres, 2000) en los cursos vinculados con la didáctica de la especialidad a los fines de contribuir con el entendimiento requerido en cuanto a la forma de trabajar en este nivel educativo. Se evidencia las dos intencionalidades en la formación de los formadores, una dirigida a su formación directa en cuanto a los propósitos de la especialidad y, la otra en función del propio trabajo escolar y de las exigencias profesionales y sociales.

Para este estudio, en lo que respecta al proceso de integración de los contenidos, los estudiantes seleccionaron un fenómeno o problema de la vida cotidiana, un tema, un tópico, una idea, una cuestión de la vida práctica y diaria de interés. Éste se constituyó en el tema central en el cual se articularían los contenidos de un área en particular y de distintas áreas del conocimiento para dar una interpretación global y unitaria de la realidad, aunque no única. Al respecto, se consideró lo propuesto por Delgado (2009, p.16) quien afirma que la realidad que vivimos es un todo coherente, en el cual se producen y reproducen los problemas y fenómenos que son irreductibles a una rigurosa visión disciplinaria. Además, se llegó al consenso de que los contenidos eran considerados, inicialmente, medios que permiten el desarrollo o la adquisición de las competencias.

A partir de esto, se eligieron los contenidos programáticos (conceptuales y procedimentales), los ejes transversales y las competencias respectivas vinculadas con el tema. Estas últimas dieron direccionalidad y orientaron el trabajo escolar, desde su diseño, implementación hasta la evaluación $y$, en conjunto, permitieron generar un propósito que orientó el proyecto educativo en un contexto específico referencial.

Adicionalmente, se incorporaron otros contenidos en función de la temática, denominados contenidos complementarios (contenidos de otros programas de estudio adaptados al nivel y grado). Asimismo, contenidos contextuales que fueron asociados a los contenidos programáticos. En relación a esto último, se generaron algunas hipótesis e interrogantes que fueron trabajadas desde el aula y comprobadas en el trabajo de campo a través de la observación, de las constataciones e inferencias que orientaron la abstracción y, en algunos casos, la generalización (García, 2000).

Se partió de varios principios que situaron el proceso de integración: (a) el aprendizaje está vinculado con la motivación y el interés, estos favorecen el desarrollo de actitudes positivas hacia el aprender; (b) cada concepto requiere de un procedimiento y, por ende, estos facilitan su adquisición; (c) la disposición para aprender y los procedimientos adecuados facilitan el desarrollo de los conceptos; (d) Es necesario el empleo de métodos complementarios que permitan el aprendizaje constructivo. 
Cada contenido conceptual se vinculó por afinidad sincrética o anastomosis (epistémica estructural, jerárquica de dependencia, organizativa, semántica, complementaria, inclusión, causación, consecuenciales, pertenencia) a través de sus contenidos procedimentales con otros contenidos procedimentales de la misma área o de las distintas áreas del conocimiento. Se infiere que esta afinidad es una conexión reticular generativa o saber compartido en un todo coherente y envolvente que se extiende desde un espacio de confluencia -bache curricular- de uno o varios nódulos pertenecientes a uno o varios campos del conocimiento, conformando así una red de conocimientos transdisciplinarios (Delgado, 2009).

Partiendo de este autor, se asumió los conceptos de nódulo conceptual, nodo o nudo y red de nódulos conceptuales en uno o varios campos del saber, así como también de los criterios de interrelación, permeabilidad e integralidad. Por otra parte, se argumentó que el principio de unicidad es integrante al conjunto natural de dichos contenidos. Por ello, se aceptó la idea originaria de Delgado (op. cit.) de que las propiedades de cualquier parte de la red se desprenden de propiedades de otras partes y el equilibrio global de sus interrelaciones determina la estructura de la totalidad de dicha red.

Siguiendo estas ideas, el campo queda permeado desde lo epistemológico, axiológico, ontológico, teleológico y metodológico al compartir sus teorías, hipótesis y hallazgos para el entendimiento y comprensión global del problema o del fenómeno. Se parte de su propia axiomática quedando subsumido por los otros, atravesado o fusionado con ellos en un tramado de relaciones que trasciende su propia individualidad en una nueva estructura.

Los contenidos procedimentales permitieron la vinculación por una de estas tres razones: (a) ser general y aplicable a cualquier disciplina, (b) requerir del procesamiento de un contenido previo de otra materia $\mathrm{o}$, (c) ser un contenido previo.

Lo que se pretende es superar la lógica lineal y reduccionista de las disciplinas aisladas para dar paso a verdaderas vinculaciones entre los conocimientos y sus formas constructivas con los saberes culturales. La idea no es desarrollar y adquirir conocimientos concretos superespecializados por el simple hecho de responder a una especialidad. Lo que se quiere es que éstos puedan ser amalgamados con otros conocimientos de diversas áreas y/o campos; entender como un conocimiento es parte de un todo coherente, las propiedades, relaciones y conexiones que se establecen entre ellos, las tramas que se configuran y, a su vez, son configuradas; es decir, las relaciones de retroacción (Morin, 2001) que caracterizan a las dinámicas que se construyen.

A continuación se presenta un ejercicio de un nódulo conceptual realizado con los diferentes grupos de estudiantes cuyo tema central fue el fenómeno del niño y la niña (Gráfico 1). El proyecto de aprendizaje se orientó a niños de segundo grado y se tituló: "La Niña Llorona". El mismo nace por la necesidad de conocer sobre vaguada que azotó 
al Estado Vargas en Venezuela para el año de 1999, sus causas y consecuencias. Aunque se hizo referencia a ambos fenómenos a través de un proceso comparativo, hubo énfasis en el fenómeno de la niña por la razón anteriormente comentada. Es necesario mencionar que una vez desarrollado algunos conceptos y teorías en clase se desarrolló el trabajo de campo en la localidad de Naiguatá, Estado Vargas.

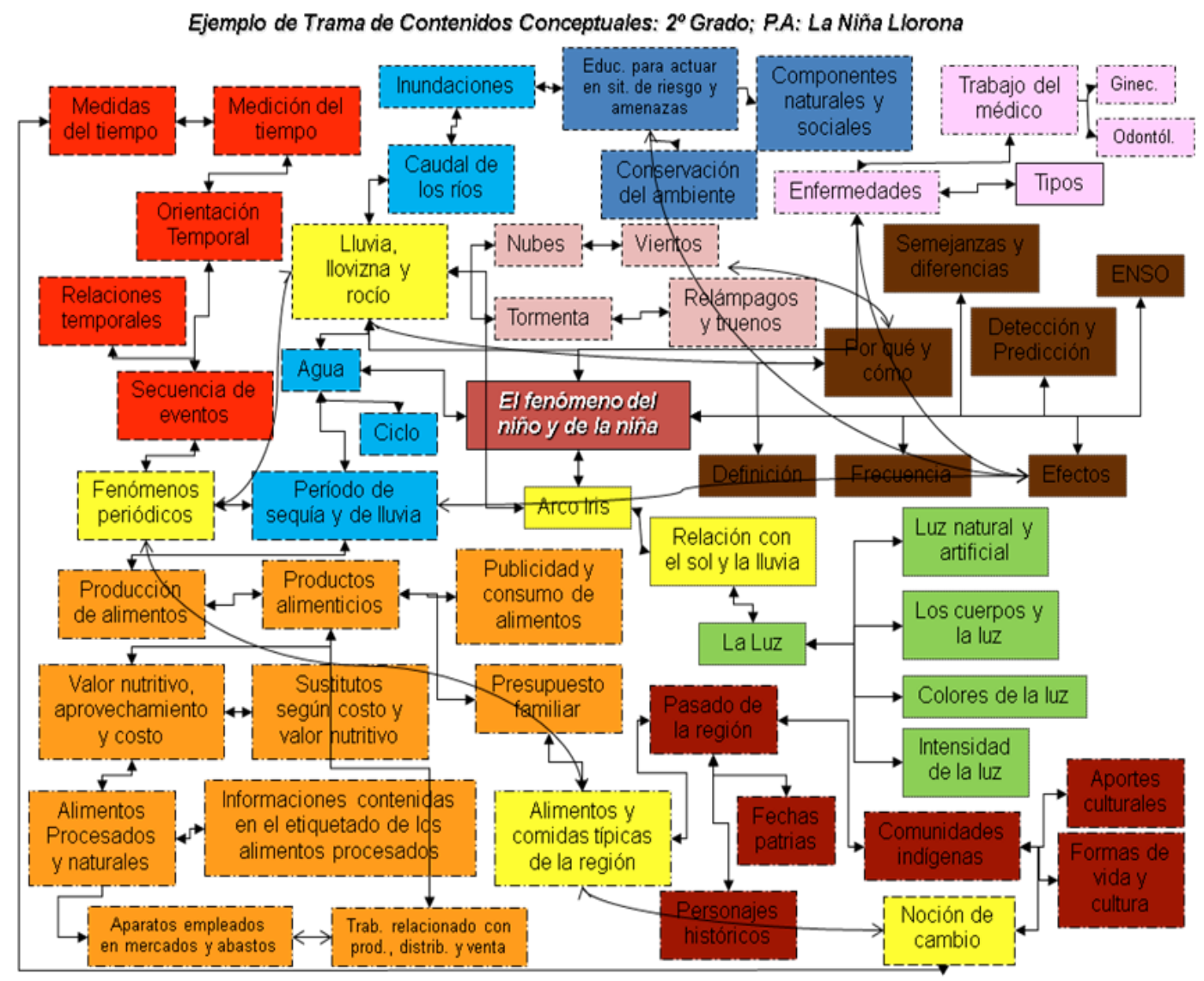

Gráfico 1. Nódulo conceptual del fenómeno del niño y la niña.

Asimismo, este nódulo ha sido empleado en distintos momentos para tratar el fenómeno ENSO en función de las condiciones climáticas en Venezuela (período de sequía o de lluvia), con algunas variantes referidas al contexto, a otros contenidos propios de cada grado y en atención de las necesidades del alumnado.

La construcción se realizó por ramales de contenidos, comenzando por la definición y caracterización del fenómeno. Luego, por conexión epistémica - estructural se incorporó el contenido referido al agua y todos sus contenidos asociados ya sean porque se referían a los hechos, objetos, conceptos, etc. Estos se ubicaron en el mismo ramal o en otro distinto que fueron organizados por afinidad jerárquica de dependencia y de pertenencia. Finalmente, se agregaron otros contenidos por afinidad de causación, consecuencial, 
inclusión y de complementariedad. De esta manera, aparecieron varios ramales que se vincularon de forma interdependiente de adentro hacia afuera.

Este proceso constructivo se caracterizó por las siguientes fases: selección, asociación, organización, aplicación de lógica e integración. Asimismo, se consideró que el aprendizaje desarrollado por los estudiantes estuvo orientado por la experiencia y la reflexión (Schön, 1987; Gimeno, 1999) que lo condujo a un proceso de abstracción y generalización (García, op. cit.).

Los estudiantes realizaron la integración de los conocimientos de las diferentes áreas del saber reconociendo la importancia de comprender el fenómeno o situación problemática ambiental como un todo sistémico y complejo; emplearon el enfoque globalizador para integrar el currículo desde la perspectiva transdisciplinaria entendiendo la realidad y sus procesos de manera integral y global.

Estudiar, comprender y aplicar la transdisciplinariedad en educación, representa un reto para el sistema educativo. Par ello, se requiere de la puesta en práctica de un currículo integrado (Torres, 2000, p.p. 113 - 124), como opción pedagógica que se sustente con la conjunción de argumentos epistemológicos y metodológicos; psicológicos y sociológicos. En este trabajo, se derivó un cuarto argumento referido a lo didáctico pedagógico. Cada uno de ellos, se presentan como fundamento de las dimensiones.

Se parte de la idea de que la transdisciplinariedad está determinada por los niveles de la realidad, la lógica del tercero incluido y la complejidad (Nicolescu, 1996). El primero hace referencia a la acción simultánea de las múltiples dimensiones que establecen relaciones, conexiones y retroacciones entre sí y con el todo (físicos, químicos, biológicos, psicológicos y socio-cultural), con sus lógicas y complejidades respectivas en el que todo está relacionado con todo (Bertalanffy, 2003). Estos niveles pueden ser estudiados desde diferentes planos con diversos enfoques y concepciones.

El segundo, la lógica del tercero incluido, se hace comprensible introduciendo la noción anterior (Nicolescu, 1996; Martínez, s.f. ${ }^{1}$; Sommerman, 2003; Carrizo, Espina y Klein, 2004; Annoni, 2004²). El todo se forma por la conjunción de las partes y de las relaciones, propiedades y diferentes dinámicas emergentes que aparecen a partir de ésta.

Se argumenta que el todo tiene cualidades o propiedades que no se encontrarían en las partes, si éstas se separan las unas de las otras; y ciertas cualidades o propiedades de las partes pueden ser inhibidas por las fuerzas que salen del todo (Morin, 2001, p. 37); mientras que otras se integran y dan las propiedades o cualidades a éste.

\footnotetext{
${ }^{1}$ http://prof.usb.ve/miguelm

${ }^{2}$ www.cybertesis.cl/tesis/uchile/2004/binotto_d/sources/binotto_d.pdf.
} 
El todo es único, distinto a cada parte por separado y existe en un nivel concreto. Varios todos interrelacionados pueden constituir una identidad de mayor nivel y complejidad que exista en otro nivel de la realidad e influyan en la dinámica de la complejidad de ese nivel. Por ejemplo, el planeta. Pero también puede observarse la parte como un todo en otro nivel distinto al todo en el que interviene y contribuye a su configuración. Por ejemplo, el fenómeno ENSO en Venezuela.

Hoy en día, cada fenómeno y problema social y natural como un "todo" debe estudiarse con la integración de varias disciplinas. Actualmente nos abruman problemáticas complejas que requieren del esfuerzo racional a escala planetaria. De allí lo imperativo de la unidad del conocimiento para comprender el mundo actual y buscar las alternativas de solución en un espacio dialógico con criterios de pluralidad conceptual y metodológica (Fuguet, 2003)

La transdisciplinariedad borra los confines disciplinarios para el tratamiento de los problemas, fusionando los saberes en redes con nódulos conceptuales; unificando el lenguaje, las teorías y concepciones, métodos y procedimientos hasta constituir sus propios polos epistemológico, ontológico, axiológico, teleológico y metodológico con una nueva visión de la naturaleza, de la realidad y del hombre. El conocimiento que se genera es pertinente al reconocer lo multidimensional de las unidades complejas (Morin, 2001). Esta perspectiva contribuye a que el sujeto realice la integración por síntesis que le permita interpretar la realidad y su complejidad.

Finalmente, haciendo referencia a la complejidad, consideramos que el tejido de trama y urdimbre está caracterizado por una red de relaciones y dinámicas que configuran al contexto (procesos, eventos, acontecimientos) que sirven de patrón para comprender la misma dinámica y la organización de los saberes. Esta complejidad es contextual, se presenta con los rasgos perturbadores de la perplejidad, es decir, de lo enredado, lo inextricable, el desorden, la ambigüedad y la incertidumbre. La realidad es multidimensional, diversa y compleja y, por lo tanto, debe ser pensada desde un espacio transdisciplinar, en permanente construcción por su carácter indeterminado, paradójico e inacabado.

En este contexto del acontecer de la humanidad se requiere un pensamiento que involucre la lógica y el afecto sin perder de vista el todo y las partes y la relación entre ellos; la conciencia de las causas y consecuencias del manejo y aplicación de la ciencia con fines pocos éticos que atenten contra la humanidad y el planeta. Así mismo, se hace necesario concebir una sola ciencia y unificar la racionalidad científica, matemática, literaria, artística, tecnológica y filosófica en una racionalidad transdisciplinar (CIRET UNESCO, 1997).

El pensamiento complejo es un pensamiento que relaciona, un arte-pensar y una estrategia del espíritu frente a la paradoja que anima el actual contexto que globaliza y 
al mismo tiempo fragmenta. Éste realiza la rearticulación de los conocimientos mediante la aplicación de sus principios generativos y estratégicos de su método, a saber: principio sistémico u organizacional, principio hologramático, principio de retroactividad, principio de recursividad, principio de autonomía - dependencia, principio dialógico y principio de reintroducción del cognoscente en todo conocimiento. A través de estos principios es preciso, tomar en cuenta la relación con el contexto (Instituto Internacional para el Pensamiento Complejo de la Universidad del Salvador, IIPC - 1997³; Morín, op. cit.).

\subsection{Dimensiones que caracterizan al trabajo de campo}

Al realizar un análisis de las experiencias desarrolladas en los cursos a partir de la aplicación del trabajo de campo y, en atención a los objetivos propuestos y de los enfoques e instrumentos empelados, se pudieron identificar algunas dimensiones caracterizadoras de esta estrategia pedagógica, a saber:

\subsubsection{Dimensión social - comunitaria.}

Se considera que el trabajo de campo es un laboratorio abierto, una oportunidad única para despertar en los estudiantes inquietudes que les permitan descubrir el sin fin de información que aparece impresa en el medio natural o social (Centro Nacional para el Mejoramiento de la Enseñanza de la Ciencia, CENAMEC, 1979; Sánchez y Godoy, 2002; Godoy y Sánchez, 2007).

Se asume que el ambiente es un sistema complejo, paradójico, dinámico, multidimensional y cambiante constituido por las interacciones entre diferentes subsistemas sociales, culturales, económicos, políticos, físicos, biológicos, religiosos, educativos. En este sentido, argumentamos que el ambiente se constituye, desde el punto de vista educativo, en un laboratorio natural, de primera mano, de experiencias enriquecedoras y favorables para el aprendizaje. Se pueden observar y estudiar variados fenómenos y problemas de la vida cotidiana, las distintas dinámicas, relaciones, propiedades, procesos, acontecimientos... que se presentan en estos.

En esta dimensión admitimos los argumentos sociológicos mencionados por Torres (2000). Él afirma que se pueden mencionar tres razones importantes para un currículo integrado. (a) La necesidad de humanizar el conocimiento; (b) la integración favorece visiones de la realidad en las que las personas aparecen como sujetos de la historia permitiendo estimular el compromiso con su realidad y obligándose a una participación más activa, responsable, crítica y eficiente; (c) la experiencia humana es de carácter holístico, el colectivo estudiantil debe aprender a analizarla y a enfrentarse con ella, saber que sus decisiones pueden estar condicionadas por múltiples razones, sus juicios y acciones de influencia van a estar mediatizadas por esa características de globalidad de la experiencia humana. 
La comprensión de los fenómenos y problemas requiere de una perspectiva que atienda lo espacial y lo histórico, lo físico y lo humano, lo natural y lo social, el pasado y el presente, lo individual y lo comunitario. En este sentido, se acepta el empleo de una didáctica que atienda la relación hombre - sociedad - naturaleza como un todo e integre a la escuela con la comunidad en el hecho de interpretar, comprender y estudiar la realidad desde lo particular, lo contextual.

El acto de poner en contacto directo al estudiante con los problemas reales de una comunidad en particular, brindó la posibilidad de conocer, discutir y reflexionar acerca de las necesidades diagnosticadas en términos de debilidades y amenazas, así como, de las fortalezas y potencialidades que permitiese el diseño de posibles alternativas de solución. Probablemente, esta experiencia contribuyó a sensibilizarlo frente a las condiciones evidenciadas y contrastadas en la comunidad. Por otra parte, se manifestó el trabajo cooperativo y colaborativo en la realización del diagnóstico situacional en el cual cada integrante fue co-responsable de una actividad o tarea en particular liderando acciones colectivas que implicaron procesos socio-afectivos.

Es necesario reconocer la importancia de la aplicación del enfoque geohistórico (Sayago, 1998; Sayago y León de F, 2000; Palencia, 2003; Ramírez y Puerta, 2008; Ancianis, Sayago y León, 2010; Sánchez y Sánchez, 2011) pues a través del empleo del diagnóstico de comunidad, el método de los conjuntos y la cartografía conceptual se construyeron y reconstruyeron algunos conocimientos sociales relacionados con las áreas de lengua y literatura (leyendas, mitos...), matemática (medidas de capacidad, longitud y peso; estadística básica...), ciencias sociales (geografía: relieve, clima, vegetación, hidrografía... y en historia: hechos resaltantes de la localidad, pasado reciente, fundación local,...), ciencias naturales (biodiversidad, flora, fauna, minerales,...), educación estética (color, formas, textura,...). Además, se establecieron las vinculaciones curriculares pertinentes de manera horizontal y vertical a través del empleo de los ejes transversales.

Al llevar a los estudiantes al medio real (social, natural; urbano, rural) se pudo constatar, a través de diferentes técnicas y procedimientos, las situaciones problemáticas de la localidad seleccionada, de tal manera que el estudiante no sólo puso en práctica algunos conocimientos previos, sino que desarrolló otros y se planteó, de manera significativa, alternativas de solución a los problemas evidenciados en dicha localidad. Godoy y Sánchez (2007) mencionan que el estudiante puede aprender acerca de los procesos, la estructura y los significados culturales de una comunidad en particular, del trabajo cooperativo y colaborativo, del manejo de instrumentos, técnicas y procedimientos variados que permitan la realización de un producto científico. Además argumentamos la posibilidad de descubrir nuevas problemáticas, relaciones y propiedades vinculadas con el tema.

${ }^{3}$ http://www.complejidad.org/penscompl.htm 


\subsubsection{Dimensión didáctica - pedagógica.}

Se refiere a la puesta en práctica de diferentes métodos, técnicas y recursos por parte del docente en función de variadas actividades con diferentes niveles de ejecución demostración y complejidad que atienden a las competencias a desarrollar y a la red conceptual transdisciplinaria elaborada en el espacio áulico. En el trabajo de campo se diseñó y empleó diversas técnicas, procedimientos y actividades con alto sentido pedagógico y de contenido práctico en espacios concretos. Un aspecto esencial lo constituye el establecimiento de objetivos claros que orienten las experiencias en función de la temática abordada en el proyecto educativo. Éstas deben caracterizarse por ser prácticas, directas, relevantes y pertinentes.

Para el nivel de Educación Primaria, se hace necesario el entendimiento global de los fenómenos y problemas naturales y sociales haciendo uso de contenidos programáticos todas las áreas del conocimiento, sus teorías y formas constructivas de abordaje para integrarlas, en el cual se promueva la investigación y sus procesos en situaciones concretas de la realidad y la puesta en práctica de los conocimientos previos en diversos contextos y actividades. Sólo se requiere de una adecuación y articulación curricular bien pensada -analizada- por parte del docente en el proyecto educativo. En el caso que nos compete, se hizo necesaria la selección adecuada de los contenidos en función del propósito de cada proyecto trabajado. Se relacionaron los contenidos de un área en particular. Esto requirió de ciertos conocimientos muy especializados vinculados, como por ejemplo, con la problemática ambiental que presenta la zona visitada, el relieve, la temperatura, la hidrografía, etc., o de conocimientos de distintas áreas tales como: la estadística, las figuras geométricas, el ritmo, la medición, la narración de poesías, la realización de dibujos, entre otros.

En este sentido, el trabajo docente debe planificarse a través de proyectos que busquen la integración en los procesos educativos en el cual se pretenda el desarrollo de competencias globalizadas relacionadas con el entendimiento de los problemas sociales y naturales, así como con la búsqueda de alternativas de solución viables y pertinentes (Delgado, 2009, p. 38).

Aunado a ello, pensamos que a partir de los argumentos psicológicos planteados por Torres, (2000) se derivan los argumentos didácticos - pedagógicos referidos a los proyectos educativos y a las estrategias de enseñanza, evaluación y aprendizaje que se ponen en práctica para el estudio concreto y contextual de los fenómenos y problemas de la vida cotidiana, empleando la investigación y otras metodologías científicas; la lectura y la producción escrita, el cine-foro como técnicas favorecedoras de los aprendizajes. Aunado a ello, el trabajo de campo como experiencia que coadyuva a la comprensión de las estructuras conceptuales, las relaciones entre los contenidos y las particularidades sociales, la construcción y reconstrucción del conocimiento desde la propia realidad, la 
contrastación teórico - práctica, el desarrollo del pensamiento crítico y la reflexión, la socialización y promoción de valores, entre otros.

Ciertamente, se deben emplear experiencias vinculadas con las artes, la literatura, la poesía, la lógica, la ciencia, la filosofía tomando en consideración al individuo en toda su plenitud y esencia, su intuición, su sentido común, su sensibilidad y su cuerpo pero también el imaginario colectivo, lo mítico, lo religioso, lo ambiental, la comunidad donde se desenvuelve y sus relaciones contextuales de manera coherente, global, trascendente e interrelacionada.

Ello implica la transformación del pensamiento lineal al pensamiento creativo, pluridimensional, multifocal, reflexivo, dinámico, flexible y capaz de construir nuevos esquemas y estructuras cognitivas, en un vaivén de aceptación de lo práctico a lo teórico, de la observación a la discusión argumentada, de lo conocido a lo desconocido, de lo incierto a lo más seguro, de la aceptación al rechazo, de lo esperado a lo inesperado, de lo racional a lo paradójico. En pocas palabras, una expansión de la mente, del modo de pensar.

Sobre la base de este último argumento, la evaluación desarrolladora, bajo los principios democrático, sistémico, gestáltico, dialéctico y ecológico, cumple un papel importante al garantizar la adquisición y el desarrollo de las competencias necesarias, basándose en los diagnósticos previos, en el uso de estrategias pedagógicas adecuadas orientadas por los procesos de autoevaluación, coevaluación y heteroevaluación, que permitan la transferencia gradual de la responsabilidad del proceso de aprendizaje. Ésta se configura en un acto de reflexión crítica continua que permite revisar las acciones emprendidas, los procesos y el desempeño en atención de las competencias globalizadas y contextualizadas.

En este sentido, las estrategias evaluativas que se propongan deben permitir comprobar hasta qué punto los estudiantes alcanzaron las competencias establecidas en comparación con el diagnóstico previo realizado sobre las condiciones personales, interpersonales y cognoscitivas.

Autores como Lossio (2004) ${ }^{4}$ afirman que el trabajo de campo es una estrategia didáctica que permita generar aprendizajes significativos en Geografía. Este autor lo diferencia como un método de enseñanza y resalta el interés particular que se debe tener en la comprensión de las ventajas de estos trabajos, pues coadyuvan a la retención, comprensión y uso activo del conocimiento. Sin embargo, se evidenció que no solo obedece a esta concepción didáctica en particular de la geografía y de las ciencias afines. El empleo de variados métodos, técnicas y actividades integradas por parte del docente para el logro de los objetivos integrales o competencias globalizadas, permitió superar la visión lineal y reducida de esta estrategia. Ello implicó una participación inicial por parte del docente con responsabilidades muy puntuales relativas a la organización y orientación 
del trabajo, seguimiento y supervisión de las tareas en términos de monitoreo, acompañamiento formativo in situ y asistencia técnica pedagógica vinculadas con las ayudas cognitivas, socioafectivas y motrices necesarias; evaluación de los procesos y productos generados. En esta etapa, hubo co-responsabilidad por parte del estudiante siendo más activa en el desarrollo, lo que permitió una construcción y reconstrucción del conocimiento y no solo un proceso de memorización y comprensión en un espacio áulico para su posterior uso durante y después del trabajo de campo. Por ejemplo, se organizaron visitas guiadas a la comunidad para comprobar, de manera directa, los problemas de inseguridad, vialidad, transporte, servicios, realización y mantenimiento de obras...

\subsubsection{Dimensión cognitiva - constructiva.}

Referida a la aplicación de procesos cognitivos, así como también, a la construcción y reconstrucción del conocimiento directamente en el sitio de trabajo, la validación de teorías y la comprobación de hipótesis. Particularmente, el trabajo de campo coadyuva a desarrollar en el alumno una actitud crítica, una conciencia social y una perspectiva más global y holística de los fenómenos y problemas sociales y naturales a través de la experiencia práctica; incrementar el gusto por la investigación y estimular el interés por los nuevos aprendizajes; poner en práctica los conocimientos previos y valorar el para qué sirven éstos en su vida cotidiana, proporcionar conocimientos sobre temas relevantes y de interés colectivo. También se pueden alcanzar competencias dirigidas al desarrollo de los procesos cognitivos básicos: observación, comparación, clasificación, definición, análisis - síntesis, memorización, inferencia y seguir instrucciones (Ríos Cabrera, 2004). Asimismo, consideramos que se desarrollan procesos cognitivos superiores, tales como: toma de decisiones y la autonomía, la resolución de problemas, el pensamiento crítico, creativo (ob. cit.) e innovador y, la evaluación.

De manera complementaria y explicativa, se asumen en esta dimensión los argumentos psicológicos planteados por Torres (2000). Están relacionados con la idiosincrasia de la psicología infantil y juvenil en la cual las necesidades e intereses del conjunto de estudiantes son aprovechadas respetando sus estructuras cognitivas y el momento para su desarrollo según el género. A ello, es necesario incorporar los estilos de aprendizajes y estrategias usadas para aprender. Otro apartado, dentro de estos argumentos, está referido al papel de la experiencia en el aprendizaje donde se incluye como un componente indispensable, la reflexión. Mediante la actividad es que se construye y reconstruye los esquemas que utiliza el individuo para comprender e intervenir sobre la realidad.

\footnotetext{
${ }^{4}$ http://www.cfh.ufsc.br/ gcn/semageo\%2025/resumo21.htm
} 
Asimismo, la importancia de los procesos en el aprendizaje en la cual se haga insistencia en destrezas básicas como la observación, comunicación, deducción, medición, clasificación, predicción, y otros procesos más complejos como son: organizar la información, tomar decisiones, analizar variable, contrastar, comparar, analizar, sintetizar, evaluar, incluyendo la capacidad de tratar y aplicar los conocimientos, estimar sus limitaciones y desarrollar los medios para superarlas.

\subsubsection{Dimensión ecológica - ambiental.}

Se refiere a la aplicación de conocimientos, métodos y técnicas propias de las ciencias sociales, al entendimiento de las variadas teorías sobre el equilibrio ambiental, la calidad de vida, los elementos témporo-espaciales, entre otros aspectos. Al poner en contacto a los estudiantes con experiencias, distanciadas del ambiente escolar y áulico, se ofrece la posibilidad de que el comportamiento se base en satisfacciones, emociones y sentimientos positivos; de responder de manera creativa, innovadora y crítica; de asumir riesgos o nuevas posibilidades, abrir el entendimiento o flexibilizar los procesos mentales, sugerir un cambio de actitud hacia el ambiente y hacia los demás, intercambiar ideas y socializar estilos, formas de pensar y valores; de incrementar su autoestima y autovalorarse en atención de sus potencialidades, desarrollo de las capacidades físicas, logar cierta independencia y sentirse seguro de sí mismo; de tomar decisiones sobre la base de la experiencia vivida, cooperar con los demás y con el trabajo en general en la consecución de metas establecidas. Aquí lo que se persigue es la promoción del desarrollo integral de la persona y un cambio de perspectiva en relación con el ambiente.

A partir de esta dimensión, el concepto de ambiente se ve fortalecido o reconstruido así como los valores implícitos vinculados con éste. El trabajo de campo permitió observar el ambiente como un sistema complejo y en total interrelación con el hombre, en el cual las acciones de uno sobre el otro recrea continuamente un bucle favorable o desfavorable sostenible y cambiable en el tiempo.

\subsubsection{Dimensión científico - académica.}

Relativa a la aplicación de conocimientos disciplinares, de teorías y métodos propios. Asimismo, hace referencia a la transformación de los conocimientos contextuales en conocimientos científicos y/o académicos. Aquí asumimos los argumentos epistemológicos y metodológicos planteados por Torres (2000) en el cual se considera que todas las ciencias poseen dos estructuras: una conceptual (sustantiva) y otra metodológica (sintáctica) que pueden ser utilizadas para investigar en otro campo diferente. La enseñanza de una ciencia integrada sirve para analizar los problemas desde el punto de vista de las diversas áreas del conocimiento. Para ello, se puede emplear cualquier forma de organización, diseño y programación. 
Particularmente, en los cursos de Metodología de la Educación Integral I y II se asumió un esquema con tres momentos importantes (Centro Nacional para el Mejoramiento de la Enseñanza de la Ciencia, CENAMEC, s.f. $\left.a^{5}\right)$ :

I. Antes del trabajo de campo: El docente debe prever algunas consideraciones entre las que destacan: (a) Seleccionar el (los) objetivo(s) o la(s) competencia(s) que se desea(n) desarrollar, reforzar o ampliar. (b) Buscar información referente al área de estudio (datos históricos, clima, suelos, vegetación, clima, entre otras que el docente considere importante). Estos se constituyen en datos secundarios obtenidos de fuentes indirectas. (c) Realizar una visita de reconocimiento para efectuar el itinerario del trabajo. Creemos necesario hacer un bloqueo de la zona (inspección previa) en la cual se observen las áreas disponibles para ejecutar las actividades, verificar los espacios de riesgos, vías de escape, préstamo de servicios, verificación de horarios de visitas habituales (depende del lugar), limitaciones de la zona. (d) Efectuar los trámites administrativos (permisología para los estudiantes ante los padres y representantes y las autoridades respectivas; en caso de ser necesario, realizar la solicitud previa del permiso para la visita; solicitud de la exoneración del pago o aplicación de la promoción por ser institución escolar; solicitud de la visita guiada; contratación o disponibilidad del transporte u otro que el docente considere). (e) Establecer un cronograma de trabajo y la distribución de las tareas por cada participante de la actividad. Además, es importante elaborar un material didáctico con breves explicaciones, un guión de preguntas, algunas orientaciones, etc. (f) Negociar las normas de disciplina y dialogar acerca del propósito de la actividad, sus beneficios e importancia. Conversar sobre las normas de seguridad y los riesgos en caso de infringir las mismas. (g) Verificar el estado de salud físico de cada participante. Considerar aquellas personas que presentan alguna enfermedad, en la cual necesariamente deben consumir medicamentos. (h) Verificar que algún miembro o acompañante posea conocimientos sobre los primeros auxilios.

También es necesario determinar el recurso didáctico requerido. Estos deben ser específicos de acuerdo a su uso, lugar de visita, la duración y objetivos que se persiguen con el trabajo de campo. Se pueden organizar en: materiales, equipos y medios. Los materiales se clasifican en: material de registro (cuaderno de notas, hojas milimetradas, pintura, lápiz y colores), material de apoyo académico (glosario de términos, investigación previa sobre el lugar de visita, mapas, cuestionarios, bitácora), material de apoyo técnico (hilo de nylon, pabilo, mecate, vara de madera de $120 \mathrm{~cm}$, lupa, linterna), material de uso personal (cédula de identidad, carnet estudiantil, medicamentos especiales, repelente, paño, botas o zapatos deportivos, mono y shorts, franelas, medias, lentes para el sol, protector solar, protector labial, producto de aseo personal, gorra, koala, morral, envase para agua, cooler o cantimplora, mudas de ropa según el número de días, ropa de dormir, sábanas, saco de dormir), material de limpieza (bolsa negra de uso variado, desinfectante, toallas húmedas), material de seguridad (tapa boca, casco, mecate), botiquín de primeros auxilios (apósitos adhesivos y no adhesivos, absorbentes y de diversos tamaños, vendas, algodón, tijera, pinzas, crema para picaduras de insectos, 
antialérgicos, agua destilada, otros), utensilios (cubiertos, vasos y platos reutilizables), suministros (alimentos, agua), material para la recolección de muestras (bolsas plásticas de cierre hermético, recipientes de plástico y/o de vidrio, tobo, pala de jardín, piqueta, navaja, bandeja de cartón), reactivos químicos (agua oxigenada, ácido clorhídrico, agua destilada).

En el caso de los equipos, los podemos clasificar en: equipo de medición (juego de geometría, cinta métrica, gotero, brújula, balanza, medidor de ph, navegador GPS, escala de Mohs, altímetro, termómetro, clinómetro, higrómetro, barómetro, anemómetro, fotómetro, curvímetro) y equipos especiales (cámara fotográfica y de video, Grabador, laptop). Existen instrumentos multifuncionales que pueden medir parámetros meteorológicos. Uno de ellos es el Multiformit que mide la temperatura, presión atmosférica, velocidad del viento y altitud (Zambrano, Sánchez, Godoy, Santiago, Lazo y Pereira, s.f.).

Los medios están referidos al uso del internet por medio de buscadores (Google, Yahoo,...) y de las redes sociales (facebook, twiter, pin, what app, skype,...) a través de teléfonos inteligentes, tablet y computadoras.

Aunado a ello, es indispensable contar con el recurso humano necesario y suficiente para el éxito del trabajo de campo. Generalmente, se puede contar con varias profesionales para que acompañen, orienten y supervisen el trabajo. Autores como Wass afirman que la proporción suficiente es de un (1) adulto por cada seis (6) niños (Wass, 1992, p.95). Sin embargo, nuestra experiencia en Venezuela nos indica que la relación es de 1 a 5. Por ejemplo, para un salón de 45 niños se puede contar con el docente del aula; el profesor de Educación Física, recreador 0 instructor de deporte y siete (7) representantes para un total de 9 adultos.

II. Durante el trabajo de campo: (a) Organizar los equipos de trabajo en función de las actividades a realizar, de los instrumentos y equipos disponibles y del espacio del que se dispone para el desarrollo de dicha actividad. (b) Realizar las observaciones cualitativas del área, en función de los objetivos que se plantea en el cronograma de actividades. (c) Realizar mediciones cuantitativas (si es el caso) y tomar las anotaciones pertinentes para su posterior análisis en clases. (d) Realizar recorridos con guía turístico (visita guiada, opcional). (e) Aplicar entrevistas o encuestas a personajes locales, historiadores, cronistas, autoridades. (f) Realizar grabaciones, tomar fotografías, levantar planos, realizar dibujos, trazar figuras y bocetos, etc. Especial importancia adquieren los datos primarios recogidos en este momento, pues posibilita la construcción y reconstrucción del conocimiento, la verificación de las hipótesis, la validación y contrastación de las teorías.

\footnotetext{
$\overline{{ }^{5} \text { http://cenamec.org.ve/html/herramientas/actividades/act05.htm }}$
} 
Durante el desarrollo del trabajo de campo, creemos necesario verificar las normas de disciplina y de seguridad, entregar el material didáctico que oriente su ejecución y brindar algunas consideraciones para la elaboración del informe final.

III. Después del trabajo de campo: (a) Realizar el análisis de los datos obtenidos durante las mediciones en campo. (b) Realizar con los estudiantes una discusión dirigida, acerca de los aspectos más relevantes desarrollados durante el trabajo, revivir la experiencia, evocar momentos y conocimientos. Además, de conocer las diferentes interpretaciones sobre los significados construidos. (c) Realizar una valoración de los nuevos aprendizajes en términos de conocimientos construidos o reconstruidos. (d) Los estudiantes deberán realizar un informe que contemple el objetivo de la actividad, el análisis de los datos, el itinerario y cronograma de trabajo, la metodología utilizada, los resultados obtenidos, las conclusiones y la importancia de la actividad realizada. Planteamos el hecho de presentar algunas actividades de cierre. Se puede realizar el intercambio de experiencias entre las diferentes secciones que hayan realizado el trabajo de campo, preparar una exposición con las fotografías y las muestras recolectadas, realizar pendones para ser mostrados en la institución y en la comunidad.

\subsubsection{Dimensión exploratoria - investigativa.}

Se refiere a los procesos de indagación previa al trabajo de campo y, por la vía de la observación y exploración in situ durante su ejecución, a la aplicación de técnicas e instrumentos bajo enfoques cuantitativos y cualitativos que permitiesen la obtención de datos e informaciones complementarias, la contrastación de hipótesis, la verificación de teorías, el empleo de parámetros estadísticos, entre otros. Esta dimensión quedó demostrada en el primer y segundo momento del trabajo de campo.

Inicialmente podemos emplear una variedad de fuentes de información: libros, referencias electrónicas, folletos editados, guías especializadas, materiales de promoción, material audiovisual, entre otros. También se pueden utilizar los archivos, censos y estudios locales. Todo este material permitirá obtener suficiente información sobre la localidad, tener una visión global sobre sus condiciones, sus límites, nivel de seguridad, vialidad, organización, funcionalidad, estructura, zonas recreativas y de esparcimiento, zonas de trabajo, tipos de visitas (guiadas, semi-guiadas, no guiadas), sitios históricos o relevantes, etc.

El hecho de que el estudiante explore su entorno; recolecte información a través de instrumentos variados; socialice la información con el resto del equipo de trabajo y del grupo en general; clasifique los objetos, plantas, animales; compare los hallazgos con los planteamientos teóricos; infiera algunos conceptos, procesos, procedimientos; generalice y valide las teorías,... se garantiza un mayor entendimiento de los fenómenos y problemas desde la propia realidad y la búsqueda de alternativas de solución; además, de la oportunidad de demostrar sus competencias adquiridas o desarrolladas 
anteriormente y consolidadas a través de la exploración in situ y/o la investigación de campo.

En cada trabajo de campo, se usaron métodos y técnicas de investigación variadas como forma de consolidar y desarrollar los conocimientos; se contrastaron las teorías en la práctica recogiendo y analizando diversas evidencias empíricas que permitieron, de manera crítica y reflexiva, la revisión de los principios fundamentales de cada teoría.

Para Martínez (s.f.) la investigación transdisciplinaria está constituida por una completa integración teorética y práctica. En ella, los participantes transcienden las propias disciplinas (o las ven sólo como complementarias) logrando crear un nuevo mapa cognitivo común sobre el problema en cuestión, es decir, llegan a compartir un marco epistémico amplio y una cierta meta-metodología que les sirven para integrar conceptualmente las diferentes orientaciones de sus análisis: postulados o principios básicos, perspectivas o enfoques, procesos metodológicos, instrumentos conceptuales, entre otros aspectos.

\subsubsection{Dimensión recreativa.}

Hace referencia a la aplicación de actividades psicomotoras y de libre esparcimiento mental en espacios no convencionales utilizando recursos del medio ambiente y contextual que permita la estimulación del proceso creativo, participativo y comunicacional. Por ejemplo, en el trabajo de campo se realizaron juegos y dinámicas, canciones, gritos y consignas. Quizás, al inicio de la actividad esta dimensión fue de fácil observación en un 40 a $45 \%$ de los estudiantes. El resto se sentían aislados y con poco ánimos de participación. Luego, de las demostraciones por parte del docente y de algunos estudiantes, se evidenció una incorporación relativamente rápida en las actividades propuestas. Muchos de ellos manifestaron sentir temor de hacer el ridículo y vergüenza de realizar la actividad de manera incorrecta. Una actividad lúdica que contribuyó a sobrellevar esta situación fue la denominada "trabajo con el error". Ello permitió un proceso gradual de integración y del reconocimiento de la existencia de la imperfección, de los incidentes, de la incertidumbre... pero también del manejo adecuado al contexto de actuación y en beneficio del ser humano y de sus aprendizajes.

\subsubsection{Dimensión valorativa.}

Hace referencia a la evaluación del proceso de aprendizaje y del desempeño individual y colectivo a través de la reflexión profunda, el diálogo y la discusión, haciendo uso de dos formas de participación: la autoevaluación y la coevaluación. Esta última dimensión quedó identificada en el segundo y tercer momento del trabajo de campo en el cual los estudiantes realizaron procesos evaluativos que implicaron la reflexión continua en la medida que ejecutaban sus actividades, la corrección o ajuste y la proposición del cambio cognitivo y la re-construcción del conocimiento en caso de ser requerido. Al final 
contrastaban la situación inicial con la situación final, generando algunas derivaciones importantes que demostraban el nuevo aprendizaje.

\subsubsection{Dimensión lingüística.}

El hecho de emplear el lenguaje propio de cada disciplina vinculada con las otras áreas del saber, permitió evidenciar un manejo del conocimiento y una apropiación de la realidad estudiada desde la misma complejidad. Estas realidades fueron designadas con términos propios conocidos y metáforas originales, que permitieron el entendimiento bajo procesos de retroalimentación continua y la redefinición de los conocimientos previos. Los estudiantes develaron las nuevas relaciones, dinámicas y prácticas para afrontar estas realidades con un lenguaje lleno de significados comunes construidos desde su propio tejido. En este sentido, fue necesaria la unificación semántica y operativa de las acepciones a través y más allá de las disciplinas. Aquí se evidencia la vinculación con las dimensiones social-comunitaria y científico-académica. Se evidenció la necesidad de superar los límites de las estructuras lingüísticas de cada disciplina, para establecer espacios conceptuales nodulares con diálogos de acción interculturales en su acepción más amplia.

\subsubsection{Dimensión axiológica formativa.}

Esta dimensión aparece en todas las etapas y fases del trabajo de campo. A partir de cada actividad se resaltaron y pusieron en práctica algunos valores tales como: la responsabilidad y co-responsabilidad, el respeto, el respeto por la diversidad, cooperación, la solidaridad, cuidado del ambiente, calidad de vida, el compañerismo, la amistad, la honestidad; así como, otras competencias vinculadas con lo afectivo actitudinal. Los procesos de reflexión profunda se incluyeron como elementos orientadores de lo axiológico en el cual se puso en evidencia la necesidad de repensar los patrones comportamentales individuales e intersubjetivos.

\section{CONCLUSIONES}

En función del objetivo propuesto en la investigación se presenta a continuación algunas derivaciones producto de razonamiento deductivo del investigador:

1. En ambos cursos se supera la visión líneal - positivista, hasta limitante, que se presenta del trabajo de campo como una simple actividad que atiende la concepción rigurosa de obtención de información en las áreas de las ciencias sociales y naturales. En atención a sus momentos didácticos, a los diferentes métodos, actividades, técnicas y procedimientos que se ponen en práctica, así como a las implicaciones contextuales y socioeducativas, curriculares, psicológicas, onto-epistémicas, teleológicas y axiológicas que se manifiestan en el desarrollo del trabajo de campo, se puede inferir que la acción 
didáctica y de aprendizaje supera esta condición al presentarse como una estrategia de integración que implica todo un trabajo transdisciplinar, creativo e innovador.

2. La realidad compleja y paradójica obliga a la utilización de análisis integrados en los cuales se consideren las múltiples dimensiones e informaciones de manera interrelacionada que permita modelar y comprender en su totalidad los fenómenos y problemas (Delgado op. cit). En este sentido, el uso de los enfoques geohistórico y globalizador permitió trabajar didácticamente desde lo contextual y local para el entendimiento del fenómeno o problema ambiental con una visión social, global y compleja, y sobre su vinculación con la calidad de vida de las personas en el contexto cultural en que vive. Este enfoque general se considera una propuesta metodológica que contribuye a establecer, en primera instancia y con carácter de complementariedad, una interrelación entre la geografía y la historia y, en segundo lugar, como punto de partida para el aprendizaje de los conocimientos de las otras áreas disciplinares con una visión integral y holística de tal manera que se entiende la realidad y la relación cultura ambiente - calidad de vida desde las teorías de la complejidad y la sistémica. Asimismo, contribuye con el establecimiento de la relación escuela - comunidad a los fines de plantear las alternativas de solución a los problemas que se le presentan a ambas organizaciones.

3. El enfoque global contribuyó de manera significativa en la relación de los contenidos de las áreas del conocimiento con los ejes transversales del diseño curricular de Educación Primaria partiendo de procesos transdisciplinarios; del conocimiento contextual - social con el conocimiento científico académico a través de procesos investigativos; a entender la relación cultura - ambiente - calidad de vida y a los problemas que surgen en este bucle. La transdisciplinariedad, constituye una estructura onto-epistémica que surge como consecuencia de la articulación total (teórica, metodológica, teleológica) entre los campos del conocimiento con dominio original, sin la existencia de límites que puedan demarcar la presencia de una especialidad o de otra, sino por lo contrario, exaltar las relaciones y dinámicas entre las partes, entre las partes y el todo, el todo y el entorno.

4. Los problemas y fenómenos ambientales que se abordaron desde la perspectiva compleja durante el trabajo de campo, permitió a los estudiantes tener una visión más amplia de estos y de presentar conscientemente soluciones viables y pertinentes concedidas con significación ética. A través de esta experiencia se fomentó el desarrollo de un pensamiento transcomplejo que permitiera integrar los saberes contextuales, académicos, filosóficos, humanísticos, científicos, tecnológicos, éticos y estéticos para el entendimiento de los fenómenos y problemas de la realidad.

5. Se puso en evidencia de que el concepto de ambiente varía de acuerdo a los individuos, los grupos humanos y, las sociedades y sus instituciones. Todo hace pensar que tal constructo depende del momento histórico y sociopolítico en que se vive, de la 
cultura y, por consiguiente, de los valores reinantes, de las prácticas, experiencias e intereses sociales, colectivos e individuales. Su significado depende del "cristal con que se mire". Esta concepción variable depende directamente de la cultura y de los diferentes grupos humanos que coexisten. Se cree que es un elemento estructurador pero también estructurado por los mismos sujetos y grupos.

6. La cultura se aprehende a través de diversos mecanismos creados para tal fin, siendo uno de ellos la educación y el sistema educativo. La educación permite la construcción de significados culturales y el sistema se encarga de dosificarlos progresivamente a través de un conjunto de experiencias adecuadas a la madurez del aprendiz y a las necesidades individuales, colectivas y sociales. En el caso particular de la Educación Primaria, creemos que esta construcción, que implica un aprendizaje, es global y situada en función de las características psicológicas del niño y adolescente, de sus potencialidades y necesidades en términos de aptitudes, de actitudes y afectividades. El aprendiz es capaz de ver la totalidad del fenómeno y/o del problema y sus significados, rara vez focaliza su atención a elementos estructurantes de los mismos. La especificidad de un proceso o dinámica y las características particulares, quizás, son observadas dentro del mismo fenómeno y no de manera aislada. Por otra parte, el hecho de poner al estudiante en contacto directo con tal laboratorio natural y/o social -ambiente-, a través de tareas prácticas que impliquen un proceso de planeamiento o preparación, descubrimiento, comprensión, ejercitación, aplicación, verificación, evaluación, entre otras, hace pensar en el verdadero valor educativo y en el significado que adquiere para el estudiante al convertirse en una experiencia que invita a aprender en los términos de la construcción y reconstrucción del conocimiento, la adquisición de valores a través de un proceso de socialización, etc. De allí la importancia de realizar proyectos educativos que atiendan la diversidad de situaciones y propongan a los estudiantes variadas experiencias altamente significativas, constructivas, globales, situadas y prácticas para ser ejecutadas en diferentes contextos, con variados niveles de ejecución y de complejidad. Una de ellas tiene que ver con el trabajo de campo que permite desde una perspectiva práctica poner en contacto al estudiante en un ambiente social y/o natural.

7. El trabajo de campo constituye por excelencia una estrategia pedagógica para la Educación Primaria que permite integrar los contenidos disciplinares y científicos, los ejes transversales y los contenidos sociales - contextuales haciendo uso de actividades para la adquisición, desarrollo y consolidación de los distintos conocimientos como un todo global y holístico. Además, favorece la identificación, el entendimiento y el abordaje de la complejidad de los problemas socioambientales para la formulación de posibles soluciones globales bajo la perspectiva de la calidad de vida. Igualmente, favorece, en los futuros docentes, la consolidación del conocimiento didáctico de las diferentes disciplinas y su integración a través de procesos transdisciplinarios. En esta estrategia se identificaron las dimensiones caracterizadoras, a saber: social - comunitaria; didáctico pedagógica; cognitiva - constructiva; ecológica - ambiental; científico - académica; exploratoria - investigativa, recreativa, valorativa, lingüística y axiológica formativa. 
8. Se contribuyó con la formación integral del docente de esta Especialidad por medio de enfoques y estrategias innovadoras tendentes a favorecer la comprensión de la realidad con una visión amplia y holística del ambiente y de la mejora de la calidad de vida en estos profesionales y de los estudiantes de primaria que estarán a su cargo en los venideros años.

\section{BIBLIOGRAFÍA}

Ancianis, E., Sayago, A. y León, E. (2010). La actividad pesquera en la Parroquia Coquivacoa Municipio Maracaibo. Material Mimeografiado. Universidad del Zulia, Facultad de Humanidades y Educación, Centro de Estudios Geográficos. Maracaibo.

Annoni, B., D. (2004). Música y Transdisciplinariedad. Santiago de Chile: Universidad de Chile. (Consultado el 31/07/2006) Disponible en: www.cybertesis.cl/tesis/uchile/2004/binotto_d/sources/binotto_d.pdf.

Bertalanffy, L. (2003). Teoría General de los Sistemas. Buenos Aires: Fondo de Cultura Económica.

Carrizo, L., Espina, M. y Klein, J. (2004). Transdisciplinariedad y Complejidad en el Análisis Social. Paris: UNESCO.

Costa, A. y Garmston, R. (1994). Cognitive Coaching; A Foundation for Renaussance Schools. Norwood, Am: Chistopher Gosdorn Publ., Inc.

Centro Nacional para el Mejoramiento de la Enseñanza de la Ciencia, CENAMEC (1979). El trabajo de campo. Boletines CENAMEC, Ciencias de la Tierra. 1(1). Pág. 1-11. CENAMEC. Caracas - Venezuela.

Centro Nacional para el Mejoramiento de la Enseñanza de la Ciencia, CENAMEC (s.f.a). El trabajo de campo para conocer el ambiente. Disponible en: http://cenamec.org.ve/html/herramientas/actividades/act05.htm

Centro Nacional para el Mejoramiento de la Enseñanza de la Ciencia, CENAMEC (s.f.b). El Diagnóstico de la Comunidad Local. Material Mimeografiado. Caracas: Ministerio de Educación y Deportes.

CIRET-UNESCO (1997). ¿Qué universidad para el mañana? Hacia una evolución transdisciplinaria de la universidad. Declaración y recomendaciones del Congreso Internacional sobre Transdisciplinariedad. Locarno (Suiza), Mayo 1997. 
Delgado, R. (2009). La integración de los saberes bajo el enfoque dialéctico globalizador: la interdisciplinariedad y transdisciplinariedad en educación. Revista Investigación y Postgrado, Vol. 24 No 3. 2009 (pp. 11-44).

Fuguet, A. (2003). Transversalidad y Transdisciplinariedad: Posibilidad de una Teorización. Investigación Doctoral. Revista Semestral de la Coordinación del Subprograma Nacional de Estudios Doctorales. 1 (1).

García, R. (2000). El conocimiento en construcción. De las formulaciones de Jean Piaget a la teoría de sistemas complejos. Gedisa. Barcelona.

Gimeno Sacristán, J. (1999). Poderes inestables de la educación. Morata. Madrid.

Godoy, I. y Sánchez, A. (2007). El trabajo de campo en la enseñanza de la geografía. Sapiens, Revista Universitaria de Investigación, Año 8, № 2, diciembre 2007, 137-146.

Instituto Internacional para el Pensamiento Complejo de la Universidad del Salvador (IIPC, 1997). ¿Qué es el pensamiento complejo y la complejidad?". (Documento en Línea consultado el 17/09/2006). Disponible en http://www.complejidad.org/penscompl.htm

Martínez M., M. (s.f). Transdisciplinariedad y Lógica Dialéctica. Un enfoque para la complejidad del mundo actual. (Consultado el 17/09/2006). Disponible en http://prof.usb.ve/miguelm

Morín, E. (2001). Los Siete Saberes Necesarios para la Educación del Futuro. Buenos Aires: UNESCO- Nueva Visión.

Nicolescu, B. (1996). La transdisciplinarité manifeste. Col. Transdisciplinarité. París: Le Rocher.

Lossio, O. (2004). El trabajo de campo en espacios urbanos como propuesta de enseñanza facilitadora de aprendizajes significativos en geografía". XXIV Semageo. Universidad Federal de Santa Catarina. Departamento de Geociencias. Resúmenes de minidiscursos. (Consultado el 08/03/13). Disponible: http://www.cfh.ufsc.br/ gcn/semageo\%2025/resumo21.htm

Palencia, Y. (2003). Praxis investigativa geohistórica para una representación cartográfica de un espacio rural. GEOENSEÑANZA, Vol.8-2003 (2), 25-39.

Ramírez, A. y Puerta, C. (2008). Geohistoria: Propuesta metodológica para la construcción del conocimiento geográfico. $V$ Encuentro Internacional: Las Transformaciones de la Profesión Docente frente a los Actuales Desafíos. Kipus, Red 
Docentes de América Latina y el Caribe. UPEL - Instituto Pedagógico Rafael Alberto Escolar Lara, Venezuela.

Ríos Cabrera, P. (2004). La Aventura de Aprender. COGNITUS, C. A. Caracas.

Sánchez, M. y Godoy, I. (2002). El trabajo de campo como estrategia metodológica para la enseñanza de las Ciencias de la Tierra. Boletín Multidisciplinario $N^{\circ} 12$. Departamento Ciencias de la Tierra. Caracas: Fundación CENAMEC.

Sánchez, C. y Sánchez, A. (2011). Aproximación al estudio geohistórico del sector noreste de la Parroquia el Paraíso, Municipio Libertador, Distrito Metropolitano de Caracas". CONHISREMI, Revista Universitaria de Investigación y Diálogo Académico, Volumen 7, Número 2, 2011, $124-167$.

Sayago, A. (1998). Propuesta cartogeohistorica de la Parroquia Cecilio Acosta Municipio Maracaibo, (Estado Zulla) como producto de la ocupación del espacio. Geoenseñanza, Vol. Especial 1998, $201-216$.

Sayago, A. y León de F., E. (2000). El Enfoque Geohistórico: Una experiencia en la praxis educativa. Geoenseñanza. Vol. 5, № 2, Universidad de Los Andes, Venezuela, 247 276.

Schön, D. (1987). La formación de profesionales reflexivos. Hacia un nuevo diseño de la enseñanza y el aprendizaje en las profesiones. Paidós. Barcelona.

Sommerman, A. (2003). Formação e Transdisciplinaridade. Uma pesquisa sobre as emergências formativas do CETRANS. Portugal: Universidade Nova de Lisboa.

Torres S., J. (2000). Globalización e interdisciplinariedad: el currículum integrado. España: Ediciones Morata, S. L.

Universidad Pedagógica Experimental Libertador - Instituto Pedagógico de Miranda José Manuel Siso Martínez (1997). Diseño Curricular de la Especialidad Educación Integral Bloque Institucional. Material Mimeografiado. Caracas: UPEL - IPM JM Siso Martínez.

Universidad Pedagógica Experimental Libertador - Instituto Pedagógico de Miranda José Manuel Siso Martínez (1999a): Programa didáctico de Metodología de Educación Integral I (MEI0714). Material Mimeografiado. Caracas: UPEL - IPM JM Siso Martínez.

Universidad Pedagógica Experimental Libertador - Instituto Pedagógico de Miranda José Manuel Siso Martínez (1999b). Programa didáctico de Metodología de Educación Integral II (MEI0724). Material Mimeografiado. Caracas: UPEL - IPM JM Siso Martínez. 
Universidad Pedagógica Experimental Libertador (2003). Manual de Trabajos de Grado de Especialización y Maestría y Tesis doctorales. FEDUPEL, Caracas.

Zambrano, J.; Sánchez, A.; Godoy, I.; Santiago, C.; Lazo, V. y Pereira, I. (s.f). Manual para el uso y manejo de mapas, G.P.S., brújula e instrumentos meteorológicos. Material mimeografiado. Caracas: Autores.

Wass, S. (1992). Salidas Escolares y trabajo de campo en la educación primaria. Madrid: Morata.

\section{René Delgado La Rosa}

Profesor en categoría de asociado a dedicación exclusiva de la Universidad Pedagógica Experimental Libertador en el Instituto Pedagógico de Miranda José Manuel Siso Martínez adscrito al Departamento de Práctica Profesional. Doctor en Educación con Estudios Postdoctorales en Educación Latinoamericana. Coordinador del Núcleo de Investigaciones de Práctica Profesional y de las Líneas de Investigación Seguimiento de los Egresados y, Formación Docente y Construcción del Conocimiento. Es investigador activo de la Línea de Investigación Laboratorio Socioeducativo (LABSOEDU) del Doctorado en Educación en el Instituto Pedagógico de Caracas y del Comité Editorial de la Revista RIEAC (Red de Investigadores en Educación en América Latina y el Caribe). Participa en Venezuela en el Programa de Estímulo para el Investigador e Innovador (PEII) en la categoría de Investigador A. 